\title{
Cardiac Ablation of SOCS3 Aggravates DOCA-Salt-Induced Hypertrophic Remodeling by Activation of Gp130- Dependent Signaling in Mice
}

\author{
Shuang Liu, ${ }^{a, b}$ Li-Xin Liu ${ }^{c} \quad$ Yun-Long Zhang ${ }^{b}$ Song Lai ${ }^{b} \quad$ Yun-Peng Xie ${ }^{b}$ \\ Nan-Nan Lic Hong-Xia Wang ${ }^{c}$ Yun-Long Xia ${ }^{b}$ Ying Liu ${ }^{b}$ Hui-Hua Lia,b \\ aDepartment of Occupational and Environmental Health, School of Public Health, Dalian Medical \\ University, Dalian, ${ }^{b}$ Department of Cardiology, Institute of Cardiovascular Diseases, First Affiliated \\ Hospital of Dalian Medical University, Dalian, 'Department of Physiology and Pathophysiology, School \\ of Basic Medical Sciences, Capital Medical University, Beijing, China
}

\section{Key Words}

Cardiac remodeling $\bullet$ Hypertrophy $\cdot$ Fibrosis $\bullet$ SOCS3 $\bullet$ DOCA-salt $\bullet$ Gp130

\begin{abstract}
Background/Aims: Cardiac remodeling is a critical pathogenetic process leading to heart failure. Suppressor of cytokine signaling-3 (SOCS3) is demonstrated as a key negative regulator of the gp130 receptor to inhibit cardiac hypertrophy. However, the role of SOCS3 in deoxycorticosterone-acetate (DOCA)-salt-induced cardiac remodeling remains unclear. Methods: Cardiac-specific SOCS3 knockout (SOCS3cKO) and wild-type (WT) C57BL/6J mice were subjected to uninephrectomy and DOCA-salt for 3 weeks. The effect of SOCS3 on cardiac remodeling and inflammation was evaluated by histological analysis. Gene and protein levels were measured by real-time PCR and immunoblotting analysis. Results: After DOCA-salt treatment, the expression of SOCS3, activation of gp130/JAK/STAT3, cardiac dysfunction and fibrosis in DOCA-salt mice were significantly elevated, which were markedly attenuated by eplerenone, a specific mineralocorticoid receptor (MR) blocker. Moreover, DOCA-salt-induced cardiac dysfunction, hypertrophy, fibrosis and inflammation were aggravated in SOCS3cKO mice, but were significantly reduced in AAV9-SOCS3-injected mice. These effects were mostly associated with activation of gp130/STAT3/AKT/ERK1/2, TGF- $\beta / \mathrm{Smad} 2 / 3$ and NF-KB signaling pathways. Conclusions: Our data demonstrate that loss of SOCS3 in cardiomyocytes promotes DOCA-salt-induced cardiac remodeling and inflammation, and it may be a novel potential therapeutic target for hypertensive heart disease.




\section{Cellular Physiology Cell Physiol Biochem 2018;47:140-150 \begin{tabular}{l|l} 
DOI: 10.1159/000489757 & Ond 2018 The Author(s). Published by S. Karger AG, Basel \\
www.karger.com/cpb
\end{tabular}}

Liu et. al.: SOCS3 Regulates DOCA-Salt-Induced Cardiac Hypertrophy

\section{Introduction}

Hypertensive cardiac remodeling is a major risk factor for cardiovascular diseases leading to heart failure. The changes include hypertension, increased cardiomyocyte size, fetal gene activation, protein synthesis and collagen deposition ultimately leading to myocardial hypertrophy and electrical conduction changes in the heart [1]. There is substantial evidence that administration of a synthetic mineralocorticoid derivative, deoxycorticosterone acetate (DOCA), in combination with a high salt intake to animals can induce cardiac remodeling, and DOCA-salt animals mimic most of the changes in human volume-overload induced hypertension [2]. However, the DOCA-salt hypertensive rat model shows a significantly decreased circulating rennin concentration and thus has been regarded as an angiotensinindependent model [3]. This may involve multiple signaling pathways, including oxidase stress, mitogen-activated protein kinases, receptor or nonreceptor-associated tyrosine kinases, inflammation and neurohumoral factors, such as endothelin, vasopressin and sympathetic nerves [2].

In this study, we presented novel evidence demonstrating that ablation of SOCS3 in cardiomyocytes significantly aggravated DOCA-salt-induced cardiac remodeling and inflammation, but overexpression of SOCS3 attenuated these pathological changes. These effects were mostly associated with activation of gp130/STAT3/AKT/ERK, TGF- $\beta / S m a d 2 / 3$ and NF-kB pathways in the hearts. Thus, our results indicate that SOCS3 plays a critical role in DOCA-salt-induced hypertrophic remodeling, and suggest that SOCS3 is a promising therapeutic target for the treatment of hypertensive cardiac diseases.

\section{Materials and Methods}

\section{Generation of cardiac-specific SOCS3 knockout mice}

SOCS $3^{\text {flox/flox }}$ mice were obtained from Jackson laboratories. To delete the SOCS3 in the myocardium, $\mathrm{SOCS}^{\text {flox/flox }}$ mice were mated with mice expressing Cre under $\alpha$-myosin heavy chain ( $\alpha$-MHC) promoter $(\alpha$-MHC-Cre). To induce Cre-dependent recombination, tamoxifen $(20 \mathrm{mg} / \mathrm{kg}$ body weight, Sigma-Aldrich) was injected intraperitoneally (i.p.) for 5 days over a 3-week duration before any further experimentation. All SOCS3 $3^{\text {flox/flox }}\left(\mathrm{WT}\right.$ ) and SOCS3 $3^{\text {flox/flox }} / \alpha$-MHC-Cre (SOCS3cKO) mice used in this study were males (10- to 12 -week-old) in a C57BL/6J background. Mice were housed under a 12 -h light-dark cycle at $18-25^{\circ} \mathrm{C}$ and received commercial mice chew and ad libitum. All procedures were performed in accordance with the protocol outlined in the Guide for the Care and Use of Laboratory Animals published by the US National Institute of Health (NIH publication No. 85-23, revised 1996) and approved by the Committee on the Ethics of Animal Experiments of Dalian Medical University.

\section{DOCA-salt treatment}

Male mice (10-12 weeks of age) were allocated randomly to 4 groups for treatment $(n=8)$ : only uninephrectomized WT mice; uninephrectomized SOCS3cKO mice; DOCA/salt-WT mice and DOCA/saltSOCS3cKO mice. Mice were anesthetized with sodium pentobarbital intraperitoneally ( $50 \mathrm{mg} / \mathrm{kg}$ ). The left kidney was then uninephrectomized. Part of animals received DOCA from subcutaneous pellets (DOCA, 2.5 $\mathrm{mg} / \mathrm{d}$; Innovative Research of America) and $0.9 \% \mathrm{NaCl}$ with drinking water for 3 weeks. Control mice were only uninephrectomized, but DOCA and saline were not given. WT mice were received eplerenone (Pfizer, Inc., New York, NY, USA), which was mixed into the diets at a dose of $1.25 \mathrm{~g} / \mathrm{kg}$ of chew). The systolic blood pressure was measured by the tail-cuff system (Softron BP98A; Softron Tokyo, Japan) 3 days before and during each of the three days of treatment as previously described [12].

Generation of adeno-associated virus vector expressing SOCS3

The mouse SOCS3 complementary DNA was constructed to adeno-associated virus (AAV) vector (pAAV-IRES-ZsGreen). The constructed AAV-SOCS3 or control vector and the packaging vectors (pHelper and pAAV-RC) were transfected into 293 AAV cells to package AAV serotypes 9 (AAV9-SOCS3 and AAV9control) (Biowit Technologies, Shenzhen, China). For in vivo transduction, $100 \mu$ l AVV9-SOCS3 or AAV9- 


\section{Cellular Physiology Cell Physiol Biochem 2018;47:140-150 \begin{tabular}{l|l} 
DOI: 10.1159/000489757 & O 2018 The Author(s). Published by S. Karger AG, Basel \\
www.karger.com/cpb
\end{tabular}}

Liu et. al.: SOCS3 Regulates DOCA-Salt-Induced Cardiac Hypertrophy

control virus particles $\left(1 \times 10^{12}\right.$ viral genomes $\left./ \mathrm{ml}\right)$ were administered to WT mice by tail vein injection. Three weeks later, mice were subjected to DOCA-salt for additional 3 weeks. Transduction efficiency of in vivo gene transfer by AAV was assessed using fluorescence microscopy and western blot analysis.

\section{Echocardiography}

Animals were anesthetized with isoflurane (3\%) and then underwent M-mode echocardiography at 21 days after DOCA-salt treatment by using a $30 \mathrm{MHz}$ probe (Vevo 770 system; VisualSonics, Toronto, Ontario, Canada) as we described previously [13-15]. The ejection fraction (EF), fractional shortening (FS), left ventricular anterior wall thickness at diastole (LVAW;d), left ventricular posterior wall thickness at diastole (LVPW;d), left ventricular inner diameter at diastole (LVID;d), left ventricular anterior wall thickness at systole (LVAW;s), and left ventricular posterior wall thickness at systole (LVPW;s) and left ventricular inner diameter at systole (LVID;s) were measured digitally on the M-mode tracings and averaged from at least 3 separate cardiac cycles. $\mathrm{EF}(\%)=100 *((\mathrm{LV}$ Vol;d $-\mathrm{LV}$ Vol;s)/LV Vol;d); LV Vol;d(ul) $=7.0 /(2.4+\mathrm{LVID} ; \mathrm{d}) *$ LVID; $\mathrm{d}^{3}$; LV Vol;s(ul)=7.0/(2.4+LVID;s)* LVID;s ${ }^{3}[16]$.

\section{Histopathology and Immunohistochemistry}

The hearts were quickly dissected out and rinsed with cool sterile saline. The hearts were fixed in $10 \%$ formalin for histological analysis. Heart sections $(5 \mu \mathrm{m})$ were stained with hematoxylin and eosin (H\&E), Masson's trichrome and Wheat germ agglutinin (WGA). Immunohistochemistry was performed with antiMac-2 antibody (Santa Cruz, CA, 1:200 dilution) and anti-SOCS3 antibody (Abcam, Cambridge, UK, 1:100 dilution). Analysis of SOCS3-positive cells, Mac-2-positive cells, myocyte cross-sectional area, fibrotic area was quantitatively performed with NIH Image 1.61 software as previously described [13-15].

\section{Quantitative real-time PCR analysis}

Total RNA was extracted from fresh mouse hearts using Trizol method (Invitrogen, Carlsbad, CA)

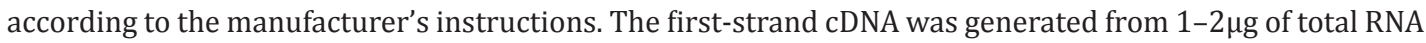
by RT Enzyme mix on a PCR Thermocycler (S1000 Thermal Cycler, Bio-Rad, USA). The mRNA expressions of atrial natriuretic peptide (ANF), B-type natriureticpeptide (BNP) and $\beta$-myosin heavy chain ( $\beta$-MHC), collagen I, collagen III, IL-1 $\beta$, IL-6, TNF- $\alpha$ and ICAM-1 were normalized to the expression of glyceraldehyde3-phosphate dehydrogenase (GAPDH) by the $\Delta \Delta \mathrm{Ct}$ method $[17,18]$.

\section{Western blot analysis}

Western blot analysis was performed as described previously $[12,13]$. Lysates were isolated from heart tissues and quantified with BCA Protein Assay according to the manufacturer's instructions. Proteins $(40-50 \mu \mathrm{g})$ were subjected to SDS-PAGE, and PVDF membrane (Bio-Rad) were probed with primary antibodies, including gp130, JAK2, p-JAK2 (Tyr1008), STAT3, p-STAT3 (Tyr705), AKT, p-AKT (Ser473), ERK1/2, p-ERK1/2 (Thr202/Tyr204), TGF- $\beta$, Smad2/3, p-Smad2/3, $\alpha$-SMA, TNF- $\alpha$, p65, p-p65 (Cell Signaling Technology, Danvers, MA, 1:800-1000 dilution) and GAPDH (Cell Signaling Technology,1:3000 dilution). Results of Western blots were quantified by the NIH Image J program.

\section{Statistical analyses}

All data were expressed as mean \pm SEM. Differences in mean values were assessed using the Student's unpaired $t$ test or two-way ANOVA for factorial design with two factors. Statistical significance was accepted at $p<0.05$.

\section{Results}

SOCS3 expression is upregulated in DOCA-salt hearts but reduced by eplerenone

To determine the functional role of SOCS3 in regulating cardiac hypertrophy and remodeling, we first examined the expression of SOCS3 in the heart. After 3 weeks of DOCAsalt treatment, SOCS3 expression was significantly increased in DOCA-salt treated heart compared with control (Fig. 1A). Moreover, immunostaining indicates that SOCS3-positive area was higher in DOCA-salt heart than in control hearts (Fig. 1B). To elucidate the mechanism by which DOCA-salt upregulates SOCS3, we co-treated mice with mineralocorticoid receptor 
(MR) inhibitor eplerenone and DOCA-salt for 3 weeks. Administration of eplerenone significantly reduced DOCA-salt-induced upregulation of gp130, p-JAK2, p-STAT3 and the downstream target SOCS3 compared with vehicle control (Fig.1C). Moreover, DOCA-salt-induced increase in cardiac performance, cardiac fibrosis and heart weight/body weight (HW/ BW) ratio was remarkably attenuated in eplerenonetreated mice compared with vehicle control (Fig. $1 \mathrm{D}-\mathrm{F})$, suggesting that DOCA-salt induced SOCS3 expression and cardiac remodeling through MRgp130 signaling.

\section{Cardiac-specific deletion of SOCS3 reduces DOCA-salt- induced cardiac performance}

To determine whether cardiac-specific deletion of SOCS3 affects cardiac remodeling and contractile performance in vivo. Echocardiography was performed. After 3 weeks of DOCA-salt treatment, no cardiac arrhythmia or sudden death was observed in SOCS3cKO mice. Systolic blood pressure was similar between WT mice and SOCS3cKO mice (Fig. 2A). However, DOCA-salt treatment resulted in increased cardiac performance as indicated by EF\% and FS\%, and this adaptive response was reduced in SOCS3cKO mice (Fig. 2B-D). In addition, DOCA-saltinduced increase of systolic and diastolic LVAW and LVPW was enhanced in SOCS3cKO mice (Fig. 2E-H). In contrast, systolic and diastolic LVID was markedly decreased in SOCS3cKO mice (Fig.2 I-J). There was no significant difference in these parameters between WT and SOCS3cKO mice after control treatment (Fig. 2C-J).

Cardiac ablation of SOCS3 accelerates DOCA-salt induced cardiac hypertrophy

DOCA-salt treatment significantly increased the heart size, LV wall thickness, ratios of HW/BW and heart weight/tibia length (HW/TL) in WT mice, and this was further

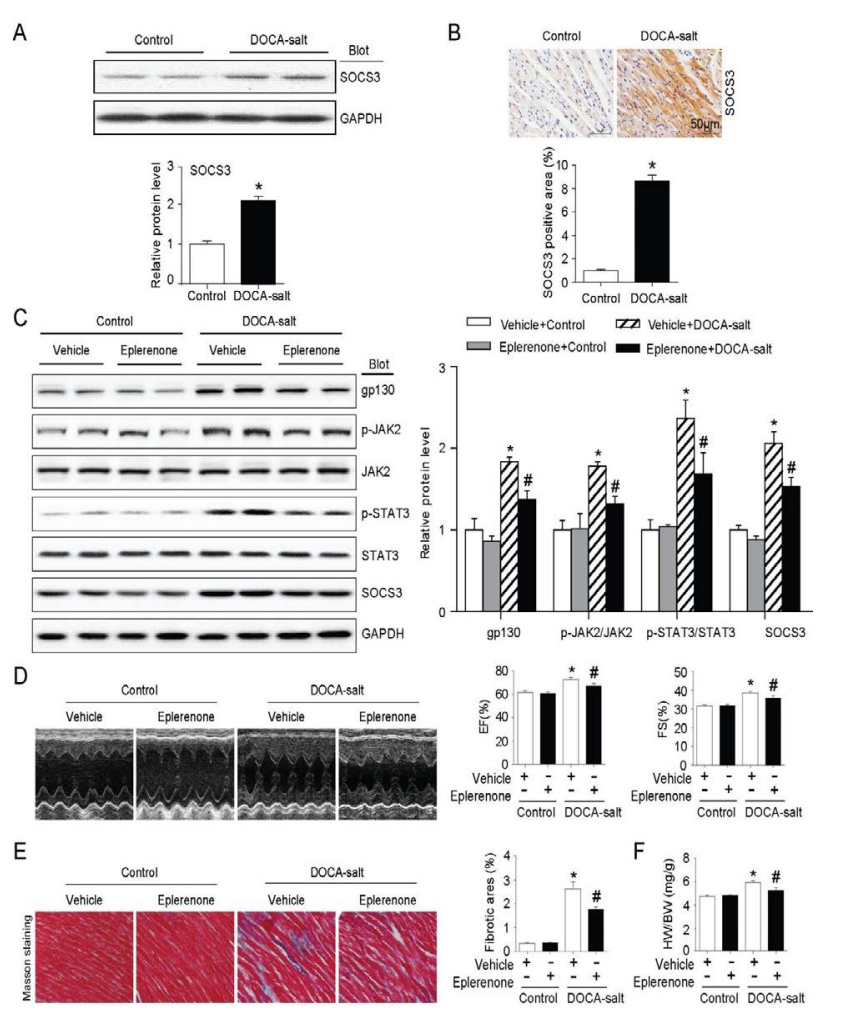

Fig. 1. DOCA-salt induced SOCS3 expression and cardiac remodeling are attenuated by eplerenone. (A) Wild-type (WT) mice were subjected to DOCA-salt for 3 weeks. The protein level of SOCS3 in the hearts was detected by Western blot analysis (upper) and quantified by densitometry values (lower). (B) Heart sections were stained with SOCS3 antibody by immunohistochemistry (upper). Scale bar $50 \mu \mathrm{m}$. Quantification of SOCS3-positive area (lower). (C) WT mice were co-treated with eplerenone and DOCA-salt for 3 weeks. Immunoblotting analyses of gp130, p-JAK2, p-STAT3 and SOCS3 in the hearts (left) and quantification of blot bands (right). (D) M-mode echocardiography of left ventricular chamber (left). Measurement of ejection fraction (EF\%) and fractional shortening (FS\%) (right, n=6). (E) Images of Masson's Trichrome staining for myocardial fibrosis (left). Magnification 200x. Quantification of the relative fibrotic area (right, $n=6$ ). (F) The ratios of heart weight to body weight $(\mathrm{HW} / \mathrm{BW})(\mathrm{n}=6)$. Data were expressed as mean \pm SEM. ${ }^{*}(\mathrm{p}<0.05)$ versus vehicle control; ${ }^{*}(\mathrm{p}<0.05)$ versus vehicle DOCA-salt. 
Fig. 2. Effect of SOCS3 deletion on blood pressure and cardiac function after DOCA-salt treatment. Wild-type (WT) and cardiac ablation of SOCS3 (SOCS3cKO) mice were subjected to DOCA-salt for 3 weeks. (A) Systolic blood pressure (SBP) was measured by mouse tail-cuff system before and after DOCA-salt treatment $(n=8)$. (B) M-mode echocardiography of left ventricular chamber. (C,D) Measurement of EF\% and FS\% (n=8). (E-J) Measurement of systolic and diastolic left ventricular anterior wall (LVAW), left ventricular posterior wall (LVPW) and left ventricular inner diameter (LVID) $(n=8)$. Data are expressed as mean \pm SEM, and $n$ represents number of animals. ${ }^{*}(\mathrm{p}<0.05)$ versus control WT; ${ }^{\#}(\mathrm{p}<0.05)$ versus DOCA-salt WT.

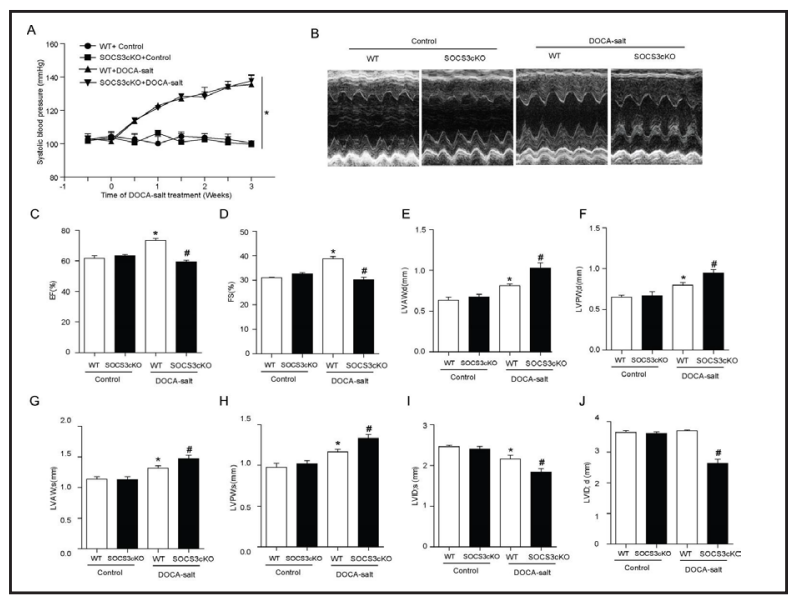

Fig. 3. Ablation of SOCS3 aggravates DOCA-salt-induced cardiac hypertrophy and activation of gp130 pathways. (A) Images of heart size (upper) and H\&E staining (lower). Scale bar $2 \mathrm{~mm}$. (B) The ratios of heart weight to body weight (HW/BW) (upper) and heart weight to tibia length (HW/ TL) (lower, $n=8$ ). (C) Images of FITClabeled wheat germ agglutinin (WGA) to detect cardiac hypertrophy (left). Scale bar $50 \mu \mathrm{m}$. Quantification of cross-sectional area of myocytes (200 cells counted per heart, right $(n=6)$. (D) qPCR analyses of ANF, BNP and $\beta$-MHC mRNA levels $(n=6)$. (E) Immunoblotting analysis of gp130, p-JAK2, JAK2, p-STAT3, STAT3, p-AKT, AKT, pERK1/2 and ERK1/2 in the heart tissue (left) Quantification of the relative protein levels (right, n=4). GAPDH was used as an internal control. Data are expressed as mean \pm SEM, and $n$ represents number of animals. $*(p<0.05)$ versus control WT; ${ }^{\#}(\mathrm{p}<0.05)$ versus DOCA-salt WT.

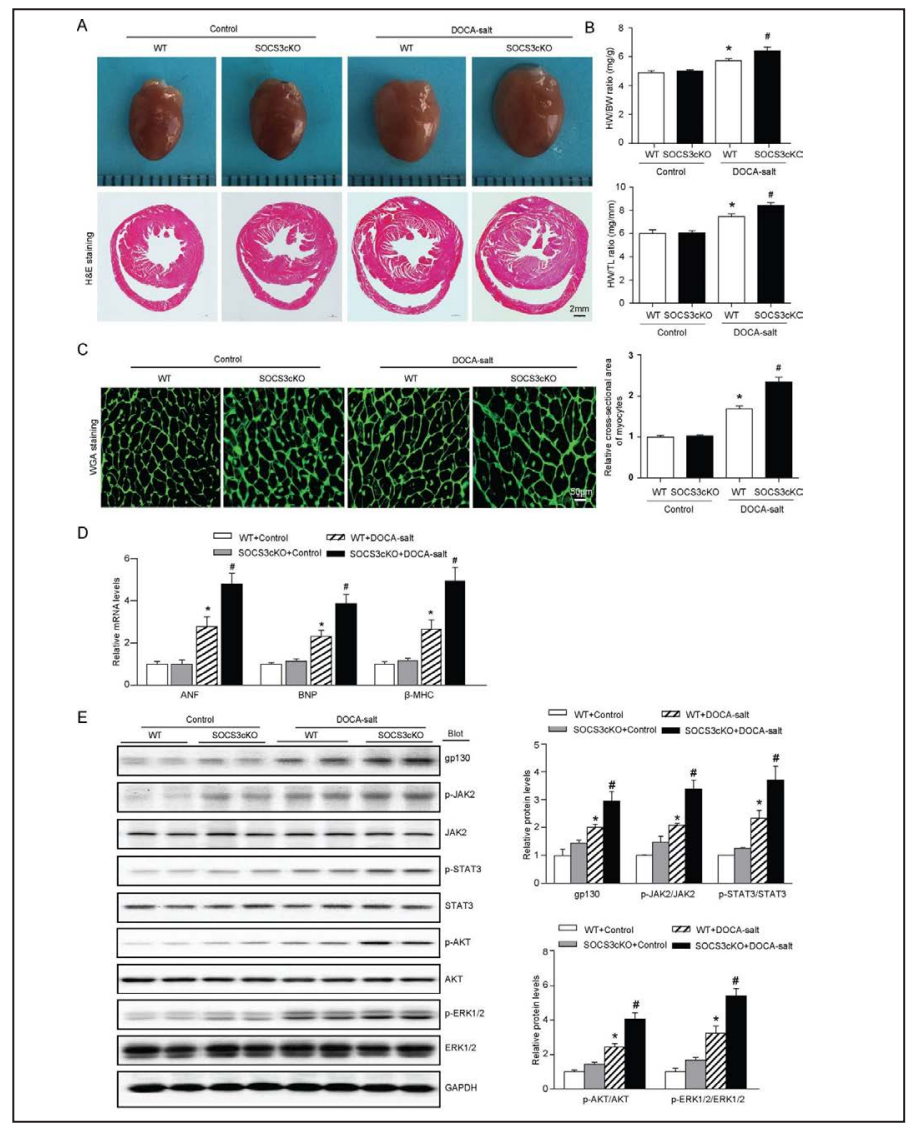

exacerbated in DOCA-salt-treated SOCS3cKO mice (Fig. 3A and 3B). Moreover, a significant increase in cardiomyocyte cross-sectional area and the expression of ANF, BNP, and $\beta$-MHC was observed in SOCS3CKO mice as compared with WT mice after DOCA-salt treatment (Fig. 3C and 3D). There was no significant difference in cardiac hypertrophy between control groups (Fig. 3A-D).

Since SOCS3 is a key negative-feedback regulator of gp130-JAK-STAT3 pathway, we then tested whether SOCS3 deficiency enhances gp130 mediated signaling. As expected, DOCAsalt induced activation of gp130, JAK2, STAT3, AKT and ERK1/2 in the hearts of WT mice was further augmented in SOCS3cKO mice (Fig. 3E). Although there was a slight increase 
in gp130 and activation of their downstream mediators under control condition, no significant statistically difference was observed between WT and SOCS3cKO mice (Fig. 3E).

Cardiac ablation
of SOCS3 enhances
DOCA-salt induced
cardiac fibrosis
Masson's trichrome staining revealed that DOCA-salt treatment significantly increased left ventricular perivascular and interstitial fibrosis as compared with WT mice in control groups, and this was markedly aggravated in DOCAsalt SOCS3cKO mice (Fig. 4A). Likewise, the increase in the mRNA expression of collagen I, collagen III and $\alpha$-SMA in the DOCA-salt WT mice was also significantly accelerated in DOCAsalt SOCS3cKO mice (Fig. 4B). No significant
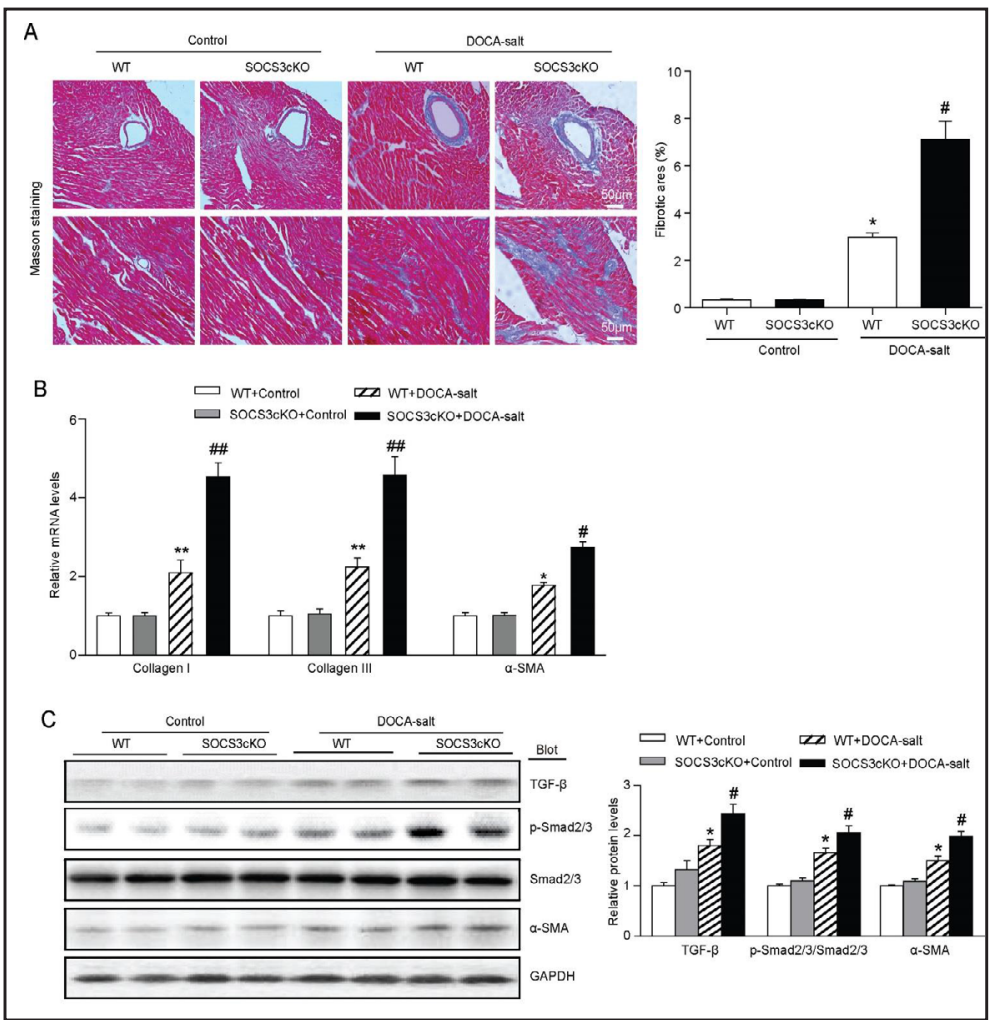

Fig. 4. Deletion of SOCS3 accelerates cardiac fibrosis in DOCA-salt hearts. (A) Images of Masson's Trichrome staining for myocardial fibrosis (left). Magnification 200x. Quantification of the relative fibrotic area (right, $n=6$ ). (B) qPCR analyses of collagen I, collagen III and $\alpha$-smooth muscle actin ( $\alpha$-SMA) levels ( $\mathrm{n}=6)$. (C) Immunoblotting analyses of TGF- $\beta$, p-Smad2/3, Smad2/3 and $\alpha$-SMA in the heart tissue (left). Quantification of the relative protein levels (right, $\mathrm{n}=4$ ). GAPDH was used as an internal control. Data are expressed as mean \pm SEM, and $\mathrm{n}$ represents number of animals. ${ }^{*}(\mathrm{p}<0.05)$ versus control WT; ${ }^{\#}(\mathrm{p}<0.05)$ versus DOCA-salt WT. difference was observed in these parameters between control groups (Fig. 4A and 4B). Moreover, DOCA-salt treatment activated TGF- $\beta$ and Smad2/3 pathways in the heart of WT mice, which was further enhanced in SOCS3cKO mice (Fig. 4C).

\section{Cardiac ablation of SOCS3 increases DOCA-salt induced cardiac inflammation}

To determine the degree of inflammatory response in the SOCS3cKO hearts, we performed H\&E and immunohistochemical staining. DOCA-salt treatment caused a marked increase in the infiltration of perivascular and interstitial proinflammatory cells, including Mac-2-positive macrophages in the DOCA-salt WT mice compared with control, and this increase was further accelerated in DOCA-salt SOCS3cKO mice (Fig. 5A and 5B). Accordingly, the mRNA expression of IL-1 $\beta$, IL- 6 , TNF- $\alpha$ and ICAM- 1 was significant higher in DOCA-salt SOCS3cKO than in DOCA-salt WT mice (Fig. 5C). Moreover, DOCA-salt-induced increase of TNF- $\alpha$ protein level and p65 phosphorylation were markedly higher in SOCS3cKO than in WT mice (Fig. 5D).

Overexpression ofSOCS3 by AAV9-SOCS3 attenuates DOCA-salt-induced cardiac remodeling

To define the effect of SOCS3 overexpression on the heart in vivo, WT mice were delivered with AAV9-SOCS3 or AAV9-control, and then subjected to DOCA-salt for 3 weeks. AAV9 injection resulted in a higher infection efficiency (Fig. 6A), and an increase of 2-fold 
SOCS3 protein in the hearts (Fig. 6B). Moreover, SOCS3 overexpression improved DOCAsalt-induced cardiac dysfunction (EF\% and FS\%) compared with AAV9-control mice (Fig. 6C). Furthermore, DOCA-salt-induced increase in the heart size, HW/BW and HW/TL ratios, cardiomyocyte cross-sectional area and fibrotic area were also attenuated in AAV9-SOCS3 mice (Fig. 7A-D). In addition, AAV9-SOCS3 injection markedly reduced gp130 level and phosphorylation of JAK2 and STAT3 after DOCA-salt treatment compared with AAV9-control

Fig. 5. Deficiency of SOCS3 promotes cardiacinflammatory response in DOCAsalt hearts. (A) Images of H\&E staining of heart sections (magnification, 200x). (B) Images of immunohistochemical staining of heart sections with Mac2 antibody (upper) (magnification, 200x). Quantification of relative Mac2-positive area (lower, n=4). (C) qPCR analysis of the mRNA expression of IL-1 $\beta$, IL-6, TNF- $\alpha$ and ICAM- 1 in the hearts $(n=6)$. (D) Immunoblotting analyses of TNF- $\alpha$, p-p65 and p65 in the heart tissue (left). Quantification of the relative protein levels (right, $n=4$ ). GAPDH was used as an internal control. Data are expressed as mean \pm SEM, and $n$ represents number of animals. $*(\mathrm{p}<0.05),{ }^{* *}(\mathrm{p}<0.01)$ versus control $\mathrm{WT} ; \quad \#(\mathrm{p}<0.05), \quad{ }^{\#}(\mathrm{p}<0.01) \quad$ versus DOCA-salt WT.

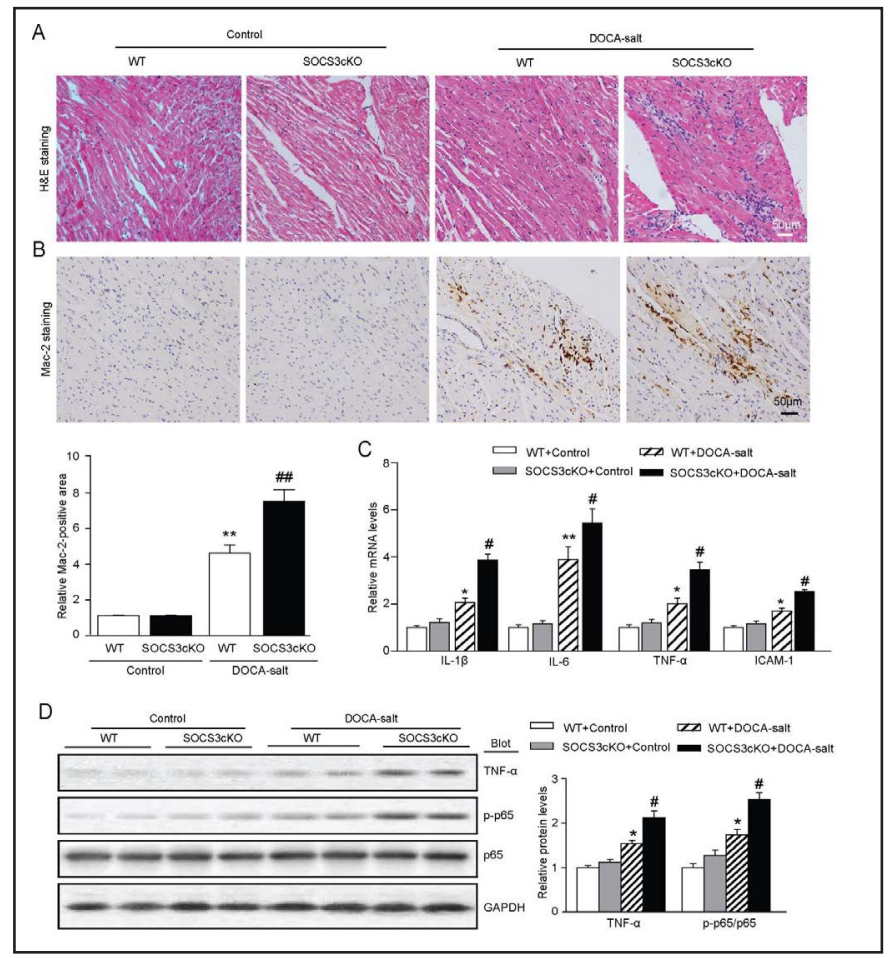

Fig. 6. Overexpression of SOCS3 improves cardiac dysfunction. WT mice injected with AAV-control or AAV9-SOCS3 were subjected to DOCA-salt for 3 weeks. (A) Fluorescence microscopic images of LV myocardium 3 weeks after tail vein AAV9-control or AAV9-SOCS3 delivery. (B) Immunoblotting analysis of SOCS3 protein levels in LV after AAV9SOCS3 or AAV9-control infection (left). Quantification of the relative protein levels (right, $n=4$ ). (C) M-mode echocardiography of LV chamber (upper). Measurement of $\mathrm{EF} \%$ and $\mathrm{FS} \%$ (lower, $\mathrm{n}=6$ ). Data are expressed as mean \pm SEM, and $n$ represents number of animals. ${ }^{*}(\mathrm{p}<0.05)$ versus AAV9control+control; $\#(\mathrm{p}<0.05)$ versus AAV9-control + DOCA-salt.

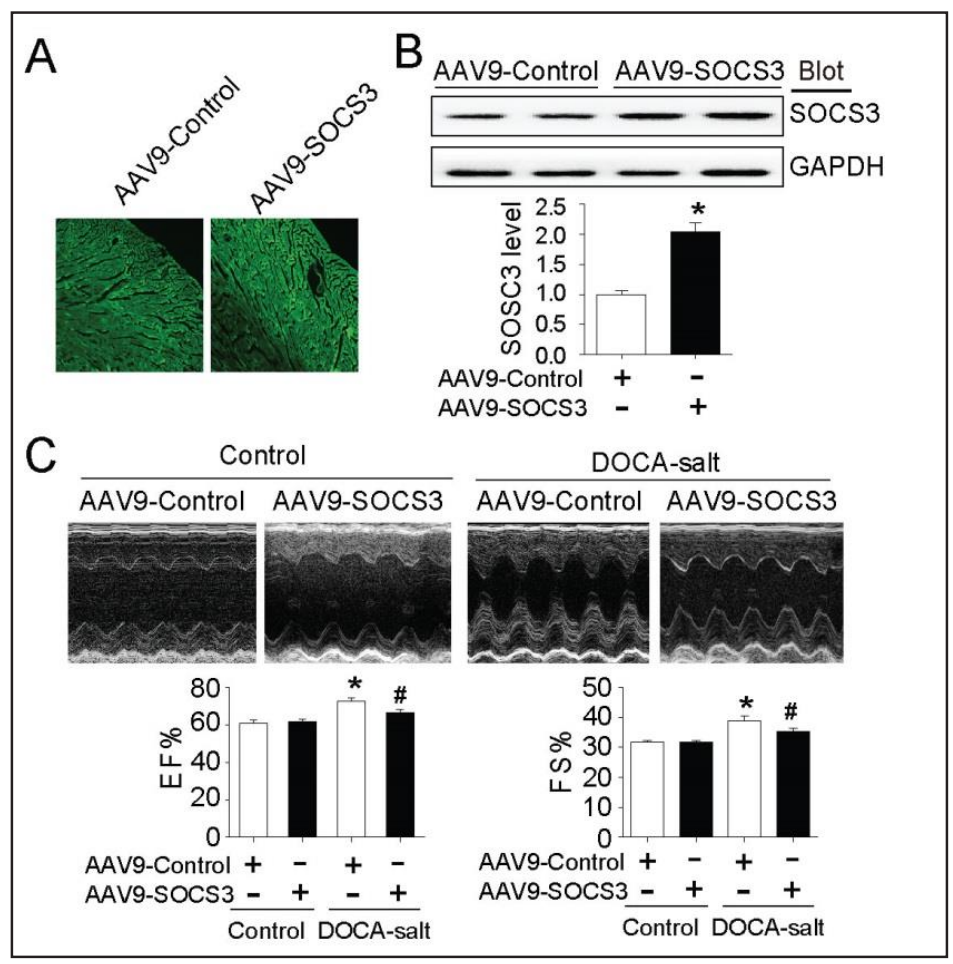


Fig. 7. Overexpression of SOCS3 inhibits cardiac remodeling. (A) Images of heart size $(n=6)$. (B) $\mathrm{HW} / \mathrm{BW}$ ratio (left) and $\mathrm{HW} / \mathrm{TL}$ ratio (right, $\mathrm{n}=6$ ). (C) Images of FITC- WGA staining for myocyte size (upper) and Masson's Trichrome staining for myocardial fibrosis (lower). (magnification, 200×). (D) Quantification of cross-sectional area of myocytes (200 cells counted per heart, $n=6$ ) and quantification of

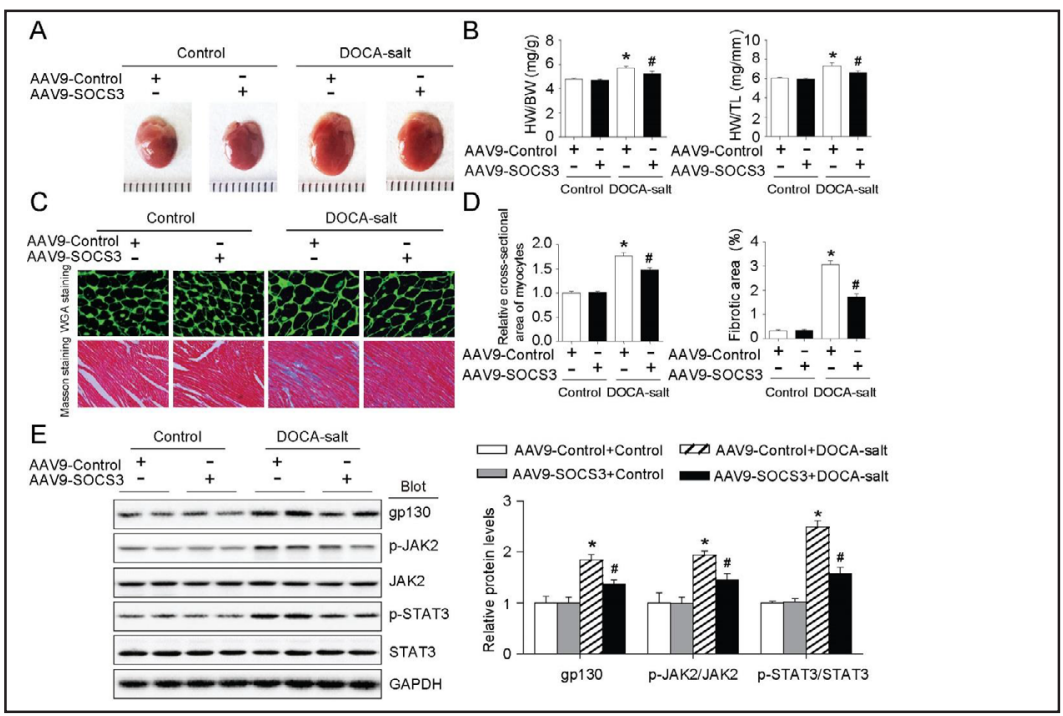
the relative fibrotic area

(right, $\mathrm{n}=6$ ). (E) Immunoblotting analysis of gp130, p-JAK2, JAK2, p-STAT3 and STAT3 in the heart tissue (left). GAPDH was used as an internal control. Quantification of the relative protein levels (right, $\mathrm{n}=4$ ). Data are expressed as mean \pm SEM, and $n$ represents number of animals. ${ }^{*}(p<0.05)$ versus AAV9-control+control; \# $(\mathrm{p}<0.05)$ versus AAV9-control + DOCA-salt.

groups (Fig. 7E). There was no significant difference in cardiac remodeling and signaling between control groups (Fig. 7A-E).

\section{Discussion}

In this study, we sought to determine the pathophysiological role of SOCS3 in DOCAsalt-induced cardiac remodeling using a SOCS3cKO animal model. We demonstrated that SOCS3 expression was significantly increased in DOCA-salt-treated heart through MR/gp130 signaling. Moreover, DOCA-salt-induced increase of cardiac remodeling were markedly aggravated in SOCS3cKO mice but attenuated in AAV9-SOCS3-injected mice. These effects were mostly associated with activation of gp130/JAK/STAT3/ERK1/2 and AKT, TGF- $\beta$ / Smad2/3 and TNF- $\alpha / N F-\kappa B$ pathways.

The SOCS proteins are identified as the target of the JAK/STAT pathway, forming a negative-feedback loop to inhibit signal propagation. They can also be induced by other signaling pathways independent of JAK/STAT signaling. Increasing evidence indicates that the list of inducers of SOCS proteins in the myocardium is growing, such as IL-6, INF- $\gamma$, TNF- $\alpha$, and angiotensin II $[4,19]$. In addition, SOCS3 is markedly induced during acute and chronic hypertrophic response following pressure overload [10]. In this study, we found that DOCA-salt-induced SOCS3 expression, activation of gp130/JAK/STAT3, cardiac dysfunction and remodeling were markedly attenuated by MR inhibitor eplerenone (Fig. 1), indicating that MR mediated DOCA-salt-induced SOCS3 expression and cardiac remodeling.

DOCA-salt rats have been reported to mimic most of the changes observed in chronic cardiac remodeling in humans including hypertension, hypertrophy, fibrosis and altered function. Furthermore, inflammation is strongly implicated in inducing cardiac hypertrophy and fibrosis in DOCA-salt animal models [20-22], which were confirmed by our findings (Fig. 2-5). Notably, inhibition of monocyte/macrophage infiltration with fasudil or tranilast markedly attenuated cardiac fibrosis in DOCA-salt rats [23, 24]. Overexpression of SOCS3 markedly reduced DOCA-salt-induced cardiac dysfunction and remodeling (Fig. 6. 7). Conversely, SOCS3 knockout aggravated DOCA-salt-induced response (Fig. 2-5). Thus, SOCS3 
plays a critical role in regulating hypertrophic remodeling and inflammation in the hearts induced by DOCA-salt stress.

It is well known that SOCS3 can directly interact with a gp130-JAK1 complex thereby regulating three major downstream pathways: JAK/STAT, Ras/MEK/ERK and PI3K/ AKT, which play different roles in the heart $[10,11]$. For example, deletion of SOCS3 in cardiomyocytes induces JAK-STAT-mediated cytokine expression, such as leukemia inhibitory factor (LIF) and G-CSF, thereby preventing acute myocardial infarction-induced left ventricular (LV) remodeling [25]. In contrast, continuous activation of gp130-STAT3 signaling aggravates inflammation and LV rupture after myocardial infarction [26]. These data suggest that gp130-JAK-STAT signaling pathway exerts anti-apoptotic role in cardiomyocytes in response to ischemic stress. Interestingly, in response to hypertrophic stress such as pressure overload, deletion of SOCS3 activates cardiac gp130 signaling including JAK-STAT, ERK1/2 and AKT results in cardiac hypertrophy and contractile dysfunction [11]. Similarly, our results also demonstrated that DOCA-salt-induced activation of gp130/JAK2/STAT3 signaling was enhanced in SOCS3CKO mice (Fig. 3), but inhibited in AAV9-SOCS3-treated hearts (Fig. 7). Overall, these data suggest that inhibition or activation of SOCS3 could be selected depending on the type of diseases. TGF- $\beta$ and its canonical downstream signaling mediator Smad are central to the development of progression of fibrosis [27]. Interestingly, IL-6/gp130 contributes to lung fibrosis via TGF- $\beta$ /Smad $2 / 3$ signaling [28]. Consistent with this study, our results further confirmed that SOCS3 deletion enhanced gp130-mediated activation of TGF- $\beta / S \operatorname{mad} 2 / 3$ signaling leading to myocardial fibrosis (Fig. 4). NF- $\kappa B$ pathway is pivotal in the production of inflammatory mediators $[29,30]$. Inhibition of NF- $\kappa B$ by Fenofibrate, an activator of PPAR- $\alpha$, significantly reduces cardiac fibrosis and improve function in DOCA-salt rat hearts [31]. In agreement with these results, our results showed that SOCS3 ablation also increased gp130-mediated activation of TNF- $\alpha$ and NF- $\kappa B$ signaling, causing increased inflammation in the hearts (Fig. 5). Collectively, cardiac deletion of SOCS3 promotes DOCA-salt-induced remodeling through gp130-mediated pathways.

In conclusion: We provide novel evidence demonstrating that SOCS3 plays a critical role in the development of DOCA-salt-induced cardiac remodeling. Ablation of SOCS3 aggravated DOCA-salt-induced cardiac dysfunction and remodeling via activation of multiple signaling pathways, whereas overexpression of SOCS3 ameliorated these effects. Thus, our data identify SOCS3 as a new potential therapeutic target for treating hypertensive heart diseases.

\section{Acknowledgements}

We thank Qing Xu for her excellent technical assistance in echocardiography. This work was supported by grants from China National Natural Science Funds (81330003, 81630009, 81500207, 31400452), International S\&T Cooperation Program of China (2014DFA31930), and Chang Jiang Scholar Program (T2011160).

\section{Disclosure Statement}

No conflict of interests exists.

\section{References}

1 Frey N, Olson EN: Cardiac hypertrophy: The good, the bad, and the ugly. Annu Rev Physiol 2003;65:45-79.

2 Iyer A, Chan V, Brown L: The doca-salt hypertensive rat as a model of cardiovascular oxidative and inflammatory stress. Curr Cardiol Rev 2003;6:291-297.

3 Kaplan NM: The current epidemic of primary aldosteronism: Causes and consequences. J Hypertens 2004;22:863-869. 


\section{Cellular Physiology Cell Physiol Biochem 2018;47:140-150 \begin{tabular}{lll} 
DOI: 10.1159/000489757 & and Biochemistry & $\begin{array}{l}\text { O } 2018 \text { The Author(s). Published by S. Karger AG, Basel } \\
\text { www.karger.com/cpb }\end{array}$ \\
\cline { 2 - 3 }
\end{tabular}}

Liu et. al.: SOCS3 Regulates DOCA-Salt-Induced Cardiac Hypertrophy

4 Yasukawa H, Nagata T, Oba T, Imaizumi T: Socs3: A novel therapeutic target for cardioprotection. JAKSTAT 2013;1:234-240.

5 Lv R, Zhao J, Lei M, Xiao D, Yu Y, Xie J: Il-33 attenuates sepsis by inhibiting il-17 receptor signaling through upregulation of socs3. Cell Physiol Biochem 2017;42:1961-1972.

6 Mahony R, Ahmed S, Diskin C, Stevenson NJ: Socs3 revisited: A broad regulator of disease, now ready for therapeutic use? Cell Mol Life Sci 2016;73:3323-3336.

-7 Zhou C, Huang J, Chen J, Lai J, Zhu F, Xu X, Wang DW: Cyp2j2-derived eets attenuated angiotensin ii-induced adventitial remodeling via reduced inflammatory response. Cell Physiol Biochem 2016;39:721-739.

-8 Marine JC, McKay C, Wang D, Topham DJ, Parganas E, Nakajima H, Pendeville H, Yasukawa H, Sasaki A, Yoshimura A, Ihle JN: Socs3 is essential in the regulation of fetal liver erythropoiesis. Cell 1999;98:617-627.

-9 Roberts AW, Robb L, Rakar S, Hartley L, Cluse L, Nicola NA, Metcalf D, Hilton DJ, Alexander WS: Placental defects and embryonic lethality in mice lacking suppressor of cytokine signaling 3. Proc Natl Acad Sci U S A 2001;98:9324-9329.

10 Yasukawa H, Hoshijima M, Gu Y, Nakamura T, Pradervand S, Hanada T, Hanakawa Y, Yoshimura A, Ross J, Jr., Chien KR: Suppressor of cytokine signaling-3 is a biomechanical stress-inducible gene that suppresses gp130-mediated cardiac myocyte hypertrophy and survival pathways. J Clin Invest 2001;108:1459-1467.

11 Yajima T, Murofushi Y, Zhou H, Park S, Housman J, Zhong ZH, Nakamura M, Machida M, Hwang KK, Gu Y, Dalton ND, Yasukawa H, Peterson KL, Knowlton KU: Absence of socs3 in the cardiomyocyte increases mortality in a gp130-dependent manner accompanied by contractile dysfunction and ventricular arrhythmias. Circulation 2011;124:2690-2701.

12 Jiang HM, Wang HX, Yang H, Zeng XJ, Tang CS, Du J, Li HH: Role for granulocyte colony stimulating factor in angiotensin ii-induced neutrophil recruitment and cardiac fibrosis in mice. Am J Hypertens 2013;26:12241233.

-13 Wang L, Li YL, Zhang CC, Cui W, Wang X, Xia Y, Du J, Li HH: Inhibition of toll-like receptor 2 reduces cardiac fibrosis by attenuating macrophage-mediated inflammation. Cardiovasc Res 2014;101:383-392.

14 Li N, Wang HX, Han QY, Li WJ, Zhang YL, Du J, Xia YL, Li HH: Activation of the cardiac proteasome promotes angiotension ii-induced hypertrophy by down-regulation of atrap. J Mol Cell Cardiol 2015;79:303-314.

15 Wang X, Wang HX, Li YL, Zhang CC, Zhou CY, Wang L, Xia YL, Du J, Li HH: Microrna let-7i negatively regulates cardiac inflammation and fibrosis. Hypertension 2015;66:776-785.

-16 Dang MQ Zhao XC, Lai S, Wang X, Wang L, Zhang YL, Liu Y, Yu XH, Li HH, Xia YL: Gene expression profile in the early stage of angiotensin ii-induced cardiac remodeling: A time series microarray study in a mouse model. Cell Physiol Biochem 2015;35:467-476.

17 Zhang JS, Zhang YL, Wang HX, Xia YL, Wang L, Jiang YN, Li HH, Liu Y: Identification of genes related to the early stage of angiotensin ii-induced acute renal injury by microarray and integrated gene network analysis. Cell Physiol Biochem 2014;34:1137-1151.

-18 Yang D, Zeng Y, Tian C, Liu J, Guo SB, Zheng YH, Li HH: Transcriptomic analysis of mild hypothermiadependent alterations during endothelial reperfusion injury. Cell Physiol Biochem 2010;25:605-614.

19 Fischer P, Hilfiker-Kleiner D: Survival pathways in hypertrophy and heart failure: The gp130-stat3 axis. Basic Res Cardiol 2007;102:279-297.

-20 Loch D, Hoey A, Brown L: Attenuation of cardiovascular remodeling in doca-salt rats by the vasopeptidase inhibitor, omapatrilat. Clin Exp Hypertens 2006;28:475-488.

-21 Brilla CG, Weber KT: Mineralocorticoid excess, dietary sodium, and myocardial fibrosis. J Lab Clin Med 1992;120:893-901.

22 Brown L, Duce B, Miric G, Sernia C: Reversal of cardiac fibrosis in deoxycorticosterone acetate-salt hypertensive rats by inhibition of the renin-angiotensin system. J Am Soc Nephrol 1999;10 Suppl 11:S143148.

23 Ishimaru K, Ueno H, Kagitani S, Takabayashi D, Takata M, Inoue H: Fasudil attenuates myocardial fibrosis in association with inhibition of monocyte/macrophage infiltration in the heart of doca/salt hypertensive rats. J Cardiovasc Pharmacol 2007;50:187-194.

24 Kagitani S, Ueno H, Hirade S, Takahashi T, Takata M, Inoue H: Tranilast attenuates myocardial fibrosis in association with suppression of monocyte/macrophage infiltration in doca/salt hypertensive rats. J Hypertens 2004;22:1007-1015. 


\section{Cellular Physiology Cell Physiol Biochem 2018:47:140-150

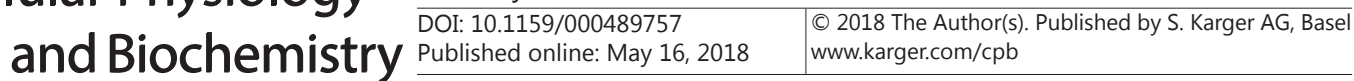 \\ Liu et. al.: SOCS3 Regulates DOCA-Salt-Induced Cardiac Hypertrophy}

25 Oba T, Yasukawa H, Hoshijima M, Sasaki K, Futamata N, Fukui D, Mawatari K, Nagata T, Kyogoku S, Ohshima H, Minami T, Nakamura K, Kang D, Yajima T, Knowlton KU, Imaizumi T: Cardiac-specific deletion of socs-3 prevents development of left ventricular remodeling after acute myocardial infarction. J Am Coll Cardiol 2012;59:838-852.

-26 Hilfiker-Kleiner D, Shukla P, Klein G, Schaefer A, Stapel B, Hoch M, Muller W, Scherr M, Theilmeier G, Ernst M, Hilfiker A, Drexler H: Continuous glycoprotein-130-mediated signal transducer and activator of transcription-3 activation promotes inflammation, left ventricular rupture, and adverse outcome in subacute myocardial infarction. Circulation 2010;122:145-155.

-27 Tang LY, Heller M, Meng Z, Yu LR, Tang Y, Zhou M, Zhang YE: Transforming growth factor-beta (tgf-beta) directly activates the jak1-stat3 axis to induce hepatic fibrosis in coordination with the smad pathway. J Biol Chem 2017;292:4302-4312.

28 Knight DA, Ernst M, Anderson GP, Moodley YP, Mutsaers SE: The role of gp130/il-6 cytokines in the development of pulmonary fibrosis: Critical determinants of disease susceptibility and progression? Pharmacol Ther 2003;99:327-338.

29 Baker RG, Hayden MS, Ghosh S: Nf-kappab, inflammation, and metabolic disease. Cell Metab 2011;13:1122.

30 Yan W, Bi HL, Liu LX, Li NN, Liu Y, Du J, Wang HX, Li HH: Knockout of immunoproteasome subunit beta2i ameliorates cardiac fibrosis and inflammation in doca/salt hypertensive mice. Biochem Biophys Res Commun 2017;490:84-90.

31 Ogata T, Miyauchi T, Sakai S, Takanashi M, Irukayama-Tomobe Y, Yamaguchi I: Myocardial fibrosis and diastolic dysfunction in deoxycorticosterone acetate-salt hypertensive rats is ameliorated by the peroxisome proliferator-activated receptor-alpha activator fenofibrate, partly by suppressing inflammatory responses associated with the nuclear factor-kappa-b pathway. J Am Coll Cardiol 2004;43:1481-1488. 